which is identical with Eq. (4.5).

Similarly, the cylindrical limit of the field perturbation in $\Phi^{0}$ is

$$
\begin{aligned}
& \frac{2 \sqrt{2} i E_{0}}{\pi} \lim _{\eta \rightarrow \infty} d(\cosh \eta-\cos \tau)^{1 / 2} \sum_{n=-\infty}^{\infty} \frac{n P_{n-1 / 2}(\cosh \eta)}{P_{n}^{0}} \\
& \times\left(Q_{n}^{0}+\frac{2 \cosh \eta_{0}}{\Pi_{n}^{0} q_{n} \sinh ^{2} \eta_{0}}\right) e^{i n \tau}=\frac{i E_{0}(\epsilon-1)}{\pi^{1 / 2}(\epsilon+1)} r \\
& \quad \times \lim _{\eta \rightarrow \infty} \sum_{n=-\infty}^{\infty} \frac{n \Gamma\left(|n|+\frac{1}{2}\right) \cosh ^{|n|+1} \eta}{|n| ! 2^{|n|-1} \cosh ^{2|n|} \eta_{0}} e^{i n \theta}
\end{aligned}
$$

by Eqs. (6.1), (6.3), (6.6), and (6.7). Hence

$\lim _{\eta \rightarrow \infty} \Phi^{0}=E_{0} r \sin \theta-\left[E_{0}(\epsilon-1) /(\epsilon+1)\right]$ $\times\left(a^{2} / r\right) \sin \theta$

which is exactly Eq. (4.6).

It is clear from Eqs. (6.9) and $(6.10)$ that the only terms in the $n$ summation that contribute to the cylindrical limit are those for which $n=-1$ and $n=1$. Since the truncation procedure of the previous section always retains these two terms, it follows that the cylindrical limit of the truncated potentials will approach the same limit as the exact solutions.
1 W. M. Hicks, Phil. Trans. 176, 161 (1884).

2 E. W. Hobson, The Theory of Spherical and Ellipsoidal Harmonics (Chelsea, New York, 1955), p. 433.

3 Higher Transcendenlal Funclions edited by A. Erdelyi (McGrawHill, New York, 1953), Vol. I.

4 L. M. Milne Thomsom, The Calculus of Finite Differences (Mac-
Millan, London 1960), p. 531

5 A proof of this result is given in Ref. 4, pp. 532-34.

6 The values of $P_{n}^{0}$ and $Q_{n}^{0}$ are taken from Tables of Associated Legendre Funclions, National Bureau of Standards (Columbia. U.P., New York, 1945).

\title{
Unitary Representations of the Homogeneous Lorentz Group in an $O(1,1) \otimes O(2)$ Basis and Some Applications to Relativistic Equations
}

\author{
E. G. Kalnins* \\ Department of Applied Mathematics, The Universily of Weslern Ontario, London, Ontario, Canada \\ (Received 6 March 1972)
}

Unitary irreducible representations of the homogeneous Lorentz group $O(3,1)$ belonging to the principal series are reduced with respect to the subgroup $O(1,1) \otimes O(2)$. As an application we determine the mixed basis matrix elements between $O(3)$ and $O(1,1) \otimes O(2)$ bases and derive recurrence relations for them. This set of functions is then used to obtain invariant expansions of solutions of the Dirac and Proca free field equations. These expansions are shown to have the correct nonrelativistic limit.

\section{INTRODUCTION}

In recent years there has been considerable interest in the unitary irreducible representations (UIR's) of the homogeneous Lorentz group in various bases. 1,2 Harmonic analysis of a scalar function in terms of the four subgroup bases [i.e., $O(3), O(2,1), E(2)$, and $O(1,1) \otimes O(2)]$ has first been given by Smorodinski and Vilenkin. ${ }^{2}$ Since this work most of the attention has been paid to the little group bases as these also play a role in the usual Poincaré invariant partial wave analysis 3,4 of scalar functions and helicity amplitudes. The properties of the reduction of $O(3,1)$ with respect to $O(1,1) \otimes O(2)$ are, however, not so well known. It is the purpose of this paper to develop these properties and indicate some possible uses. The content of the paper is arranged as follows. In Sec. 1 we collect the pertinent facts concerning $S L(2, C)$ [the covering group of $O(3,1)$, its Lie algebra and UIR's. In Sec. 2 we carry out the reduction of the principal series of $S L(2, C)$ with respect to $D(1,1) \otimes D(2)$ (see Sec. 2) the universal covering group of $O(1,1) \otimes O(2)$. The action of the infinitesimal generators of the Lie algebra in such a basis is also determined. In Sec. 3 we develop the expansion of a single particle helicity state in terms of mixed basis matrix elements. An explicit expression for these matrix elements is obtained for the first time. In Sec. 4 we derive recurrence relations for these mixed basis matrix elements, which are used in Sec. 5 to develop invariant expansions of solutions of the free field Proca and Dirac equations. Finally in Sec. 6 the nonrelativistic limit of these solutions is obtained.

\section{RESUMĖ OF $S L(2, C)$ AND TIS UIR'S}

The group $S L(2, C)^{5}$ is the universal covering group of the homogeneous Lorentz group $O(3,1)$. The elements of $S L(2, C)$ are the unimodular complex matrices in two dimensions

$$
g=\left(\begin{array}{cc}
\alpha & \beta \\
\gamma & \delta
\end{array}\right), \quad \alpha \delta-\beta \gamma=1
$$

The subgroup $S U(2)$ consists of all unitary unimodular matrices of the form

$$
\left(-\frac{\alpha}{\beta} \frac{\beta}{\alpha}\right), \quad|\alpha|^{2}+|\beta|^{2}=1 .
$$

$S U(2)$ is of course the covering group of $O(3)$ the real orthogonal group in three dimensions. The covering group of $O(1,1) \otimes O(2)$ is denoted by $D(1,1) \otimes D(2)$ and consists of all diagonal unimodular matrices:

$$
\left(\begin{array}{ll}
\alpha & 0 \\
0 & \beta
\end{array}\right), \quad \alpha \beta=1
$$

Note: $D(2)$ is the set of all diagonal matrices of the form

$$
R(\psi)=\left(\begin{array}{ll}
e^{i \psi / 2} & 0 \\
0 & e^{-i \psi / 2}
\end{array}\right), \quad 0 \leq \psi \leq 2 \pi,
$$

such that to each rotation in the plane of the group $O(2)$ there corresponds the matrices $\pm R(\psi)$. This is just the usual two to one homomorphism between an orthogonal group and its spinor group. Similar remarks apply to $D(1,1)$ the set of matrices 


$$
\left.\pm\left(\begin{array}{ll}
e^{a / 2-0} \\
0 & e^{-a / 2}
\end{array}\right), \quad-\infty<a<+\infty\right] \text {. }
$$

The Lie algebra of $S L(2, C)$ is six dimensional, being spanned by the generators $M_{i}, N_{i}(i=1,2,3)$ which satisfy the commutation relations

$$
\begin{array}{r}
{\left[M_{i}, M_{j}\right]=\epsilon_{i j k} M_{k}, \quad\left[M_{i}, N_{j}\right]=\epsilon_{i j k} N_{k},} \\
{\left[N_{i}, N_{j}\right]=-\epsilon_{i j k} M_{k} .}
\end{array}
$$

There are two independent Casimir invariants of $S L(2, C)$ which label each irreducible representation. They are

$$
K_{1}=\mathbf{M}^{2}-\mathbf{N}^{2}, \quad K_{2}=\mathbf{M} \cdot \mathbf{N} .
$$

The Casimir invariant of $S U(2)$ is well known to be $\mathbf{M}^{2}$. Each inequivalent UIR of $S U(2)$ is labeled by the eigenvalue $j$, where

$$
\mathbf{M}^{2}=-j(j+1), \quad j=0, \frac{1}{2}, 1, \frac{3}{2}, \cdots .
$$

Each UIR for given $j$ is $(2 j+1)$-dimensional and the spectl $d \mathrm{~m}$. of $M_{3}$ in it is

$$
M_{3}=-j,-j+1, \ldots, j-1, j .
$$

A UIR of $D(1,1) \otimes D(2)$ is labeled by the two eigenvalues of $M_{3}$ and $N_{3},\{m, \tau\}$ where

$$
-\infty<\tau<+\infty, \quad m=0, \pm \frac{1}{2}, \pm 1, \pm \frac{3}{2}, \cdots .
$$

It is easy to see that each such UIR is onedimensional.

We now give the spectrum of the Casimir operators $K_{1}, K_{2}$ corresponding to the principal series $\left\{j_{0}, \rho\right\}$ of $S L(2, C)$ together with the spectrum of $j$ values of the UIR's of $S U(2)$ that appear in each such UIR of $S L(2, C)$. For the principal series

$$
\begin{aligned}
K_{1}=1+\rho^{2}-j_{0}^{2}, \quad K_{2}=-\rho j_{0}, \\
j_{0}=0, \frac{1}{2}, 1, \frac{3}{2}, \ldots, \quad-\infty<\rho<+\infty,
\end{aligned}
$$

and the spectrum of $j$ values is

$$
j=j_{0}, j_{0}+1 \cdots \text {. }
$$

The other set of UIR's of $S L(2, C)$ belong to the complementary series which we write as $\{0, i \rho\}$, where

$$
\begin{aligned}
& K_{1}=1-\rho^{2}, \quad K_{2}=0, \quad 0<\rho<1, \\
& j_{0}=0,1,2, \cdots .
\end{aligned}
$$

This set of UIR's does not figure in the completeness relation ${ }^{5}$ of $S L(2, C)$ and so will not be considered subsequently.

Finally in this section we give the formulas for the action of the generators $M_{i}, N_{i}$ on an $S U(2)$ basis of the principal series

$$
\begin{aligned}
& M_{3}|j, m\rangle=m|j, m\rangle, \\
& M_{+}|j, m\rangle=-i \alpha_{\lambda+1}^{j}|j, m+1\rangle, \\
& M_{-}|j, m\rangle=-i \alpha_{\lambda}^{j}|j, m-1\rangle, \\
& N_{3}|j, m\rangle=-i \sqrt{ }\left[j^{2}-m^{2}\right] c_{j}|j-1, m\rangle
\end{aligned}
$$

$$
\begin{aligned}
& +i A_{j} m|j, m\rangle \\
& +i C_{j+1} \sqrt{ }\left[(j+1)^{2}-m^{2}\right]|j+1, m\rangle, \\
& N_{+}|j, m\rangle=-i C_{j} \sqrt{ }[(j-m)(j-m-1)]|j-1, m+1\rangle \\
& \quad+i A_{j} \sqrt{ }[(j-m)(j+m+1)]|j, m+1\rangle \\
& \quad-i C_{j+1} \sqrt{ }[(j+m+1)(j+m+2)]|j+1, m+1\rangle, \\
& N_{-}|j, m\rangle=i C_{j} \sqrt{ }[(j+m)(j+m-1)]|j-1, m-1\rangle \\
& +i A_{j} \sqrt{ }[(j+m)(j-m+1)]|j, m-1\rangle \\
& +i C_{j+1} \sqrt{ }[(j-m+1)(j-m+2)]|j+1, m-1\rangle,
\end{aligned}
$$

where

$$
A_{j}=\frac{-j_{0} \rho}{j(j+1)}, \quad C_{j}=\frac{i}{j}\left(\frac{\left(j^{2}-j_{0}^{2}\right)\left(j^{2}+\rho^{2}\right)}{4 j^{2}-1}\right)^{1 / 2},
$$

$m=-j,-j+1, \ldots, j, \quad j=j_{0}, j_{0}+1, \ldots$,

and $|j, m\rangle$ is an abbreviation for $\left|\rho j_{0} ; j m\right\rangle$ :

$$
\alpha_{\lambda}^{j}=\sqrt{ }[(j(j+1)-\lambda(\lambda-1)] .
$$

\section{REDUCTION OF THE PRINCIPAL SERIES OF $S L(2, C)$ UNDER $O(1,1) \otimes O(2)$}

As is well known ${ }^{5}$ the principal series of $S L(2, C)$ is realized via unitary transformations in a Hilbert space $H$ of square integrable functions in a certain domain. The elements of $H$ are specified by functions $f(z)$ of a single complex variable $z$ varying over the entire complex plane. (This specification is only possible up to sets of measure zero.) The scalar product and norm are given by

$$
\begin{aligned}
(f, h)=\int_{-\infty}^{\infty} d x \int_{-\infty}^{\infty} d y \overline{f(z)} h(z), \quad z=x+i y, \\
\|f\|=(f, f)^{1 / 2}<\infty .
\end{aligned}
$$

In the UIR $\left\{j_{0}, \rho\right\}$ of the principal series, the unitary operator $U(g)$ representing the group element $g$ acts on $f(z)$ in the following way:

$$
\begin{aligned}
{[U(g) f](z)=(\delta+\beta z)^{j_{0}-1+i \rho}(\bar{\delta}+\overline{\beta z})^{-j_{0}-1+i \rho} } & \\
& \times f[(\alpha z+\gamma) /(\beta z+\delta)]
\end{aligned}
$$

This realization is not the most convenient one for our purposes. In order to realize the principal series in a $D(1,1) \otimes D(2)$ basis, we make the following transformation:

$$
\begin{aligned}
& e^{a}=\left(x^{2}+y^{2}\right)^{1 / 2}, \quad \tan \phi=y / x, \\
&-\infty \leq a \leq+\infty, \quad 0 \leq \phi \leq 2 \pi .
\end{aligned}
$$

Instead of specifying an element of $H$ by $f(z)$ we specify it by the new function

$$
\tilde{f}(a, \phi)=e^{-i j_{0} \phi} e^{a(1-i \rho)} f(z) .
$$

With this indentification the scalar product can be written

$$
(f, h)=\int_{0}^{2 \pi} d \phi \int_{-\infty}^{\infty} d a \overline{\bar{f}(a, \phi)} \tilde{h}(a, \phi) .
$$

The generators $M_{i}, N_{i}$ acting on the $\tilde{f}(a, \phi)$ functions can be expressed as differential operators acting on $a$ and $\phi$ as 


$$
\begin{aligned}
M_{1}= & j_{0} \cosh a \cos \phi-(\rho+i) \sinh a \sin \phi \\
& +i\left(\sinh a \cos \phi \frac{\partial}{\partial \phi}-\cosh a \sin \phi \frac{\partial}{\partial a}\right), \\
M_{3}= & -i \frac{\partial}{\partial \phi}, \\
N_{1}= & j_{0} \sinh a \sin \phi+(\rho+i) \cosh a \cos \phi \\
& +i\left(\cosh a \sin \phi \frac{\partial}{\partial \phi}+\sinh a \cos \phi \frac{\partial}{\partial a}\right), \\
N_{3}= & -i \frac{\partial}{\partial a} .
\end{aligned}
$$

The operators $M_{2}, N_{2}$ can be obtained from the expressions for $M_{1}$ and $N_{1}$, respectively, via the substitution $\phi \rightarrow-\frac{1}{2} \pi+\phi$. The principal series of $S L(2, C)$ is now realized as the set of functions $\tilde{f}(a, \phi)$ on the domain $(-\infty,+\infty) \otimes[0,2 \pi]$ which satisfy

$$
(f, f)=\int_{0}^{2 \pi} d \phi \int_{-\infty}^{\infty} d a|\tilde{f}(a, \phi)|^{2}<\infty .
$$

The two Casimir invariants of $O(1,1) \otimes O(2)$ are $N_{3}$ and $M_{3}$, so that the simultaneous eigenfunctions of $N_{3}$ and $M_{3}$ in this realization are

$$
\Psi_{\tau m}=[1 /(2 \pi)] e^{i \tau a} e^{i m \phi},
$$

where

$$
\begin{array}{ll}
N_{3} \Psi_{\tau m}=\tau \Psi_{\tau m}, \quad & M_{3} \Psi_{\tau m}=m \Psi_{\tau m}, \\
& \left(\Psi_{\tau^{\prime} m}, \Psi_{\tau m}\right)=\delta_{m, m} \delta\left(\tau^{\prime}-\tau\right) ;
\end{array}
$$

so together with the completeness relations ${ }^{6}$

$$
\begin{aligned}
& \frac{1}{2 \pi} \int_{-\infty}^{\infty} e^{i\left(\alpha^{\prime}-a\right)_{i}} d \tau=\delta\left(a^{\prime}-a\right) \\
& \frac{1}{2 \pi} \sum_{p=-\infty}^{\infty} e^{i p\left(\phi-\phi^{\prime}\right)}=\sum_{n=-\infty}^{\infty} \delta\left(\phi-\phi^{\prime}-2 \pi n\right),
\end{aligned}
$$

we get the following result.

Each UIR $\left\{j_{0}, \rho\right\}$ of the principal series of $S L(2, C)$ contains each UIR $\{m, \tau\}$ of $D(1,1) \otimes D(2)$ exactly once, provided

$$
m=j_{0}, j_{0} \pm 1, j_{0} \pm 2, \cdots \text {. }
$$

Thus each $\tilde{f} \in H$ can be expanded in terms of the eigenfunctions $\Psi_{\tau m}$ according to

$$
\begin{aligned}
\tilde{f}=\sum_{m=-\infty}^{\infty} \int_{-\infty}^{\infty} d \tau f_{m}(\tau) \Psi_{\tau m}, & \\
& f_{m}(\tau)=\int_{0}^{2 \pi} d \phi \int_{-\infty}^{\infty} d a \tilde{f} \tilde{\Psi_{\tau m}} .
\end{aligned}
$$

Finally in this section we calculate the action of the generators $M_{ \pm}, N_{ \pm}$on the $\Psi_{\tau m}$ basis

$$
\begin{aligned}
M_{ \pm} \Psi_{\tau m}= & \frac{1}{2}\left(j_{0} \pm i \rho \mp i \tau \mp 1-m\right) \Psi_{\tau-i, m \pm 1} \\
& +\frac{1}{2}\left(j_{0} \mp i \rho \mp i \tau \pm 1+m\right) \Psi_{\tau+i, m \pm 1}, \\
N_{ \pm} \Psi_{\tau m}= & \frac{1}{2}\left(\mp i j_{0}+\rho-\tau+i \pm i m\right) \Psi_{\tau-i, m \pm 1} \\
& +\frac{1}{2}\left( \pm i j_{0}+\rho+\tau+i \pm i m\right) \Psi_{\tau+i, m \pm 1}, \\
N_{ \pm}= & N_{1} \pm i N_{2}, M_{ \pm}=M_{1} \pm i M_{2} ;
\end{aligned}
$$

The action of $M_{3}$ and $N_{3}$ already having been given in Eq. (2.9).

\section{CALCULATION OF THE MIXED BASIS MATRIX ELEMENTS AND STNGLE PARTICLE HELICITY STATES}

In this section we construct relativistic functions with helicity for nonvanishing mass which are at the same time basis functions of a UIR $\left\{j_{0}, \rho\right\}$ of the homogeneous Lorentz group $O(3,1)$ realized on the upper sheet of a double sheeted hyperboloid. In order to do this we use the method of Integral geometry. ${ }^{2,7}$ In this method 7,8 a one-particle state of spin $s$, helicity $\lambda$, and four velocity $u$, denoted by $|u, s, \lambda\rangle$, is expressed in terms of a function on the light cone $\Phi_{j_{0} \rho}(\xi)$ via the relation

$$
\begin{aligned}
|u, s, \lambda\rangle=\frac{1}{2(2 \pi)^{3}} & \sum_{j_{n}=-s}^{s} \int_{-\infty}^{\infty} d \rho\left(\rho^{2}+j_{0}^{2}\right) \\
& \times \int_{\Gamma}[u, \xi]^{-1-i \rho} D_{\lambda j_{0}}^{s}(R) \Phi_{j_{0} \rho}(\xi) d^{2} \xi,
\end{aligned}
$$

where $\Gamma$ is the integration path on the light cone, $d^{2} \xi$ the invariant measure on the cone, and $[u, \xi]$ the usual Lorentz scalar product

$$
[u, \xi]=u_{0} \xi_{0}-\mathbf{u} \cdot \xi .
$$

The rotation specified by $D_{\lambda j}(R)$ is the rotation necessary to account for the requantization of the helicity component from the direction $\xi$ to that of $u$. The parametrization of the four velocity $u$ in the coordinate system of interest (the $C$ system or cylindrical system ${ }^{2}$ ) is

$u=(\cosh a \cosh b, \sinh a \cos \psi, \sinh a \sin \psi, \cosh a \sinh b)$,

and the 4 -vector $\xi$ is parametrized by

$$
\xi=e^{c}(\cosh \beta, \cos \phi, \sin \phi, \sinh \beta) .
$$

The choice of $\Gamma$ for the $C$ system is $\xi_{0}^{2}-\xi_{3}^{2}=1$, and the consequent invariant measure is $d^{2} \xi=d \phi d \beta$.

In the realization on the cone the generators of the Lorentz group corresponding to a "photon" of discrete helicity $\lambda$ are ${ }^{9}$

$$
\begin{aligned}
& M_{1}=-i(\xi, \nabla)_{1}+\lambda\left[\xi_{1} /\left(\xi_{0}+\xi_{3}\right)\right], \\
& M_{2}=-i(\xi, \nabla)_{2}+\lambda\left[\xi_{2} /\left(\xi_{0}+\xi_{3}\right)\right], \\
& M_{3}=-i(\xi, \nabla)_{3}+\lambda, \\
& N_{1}=-i \xi_{0} \frac{\partial}{\partial \xi_{1}}-\lambda \frac{\xi_{2}}{\xi_{0}+\xi_{3}}, \\
& N_{2}=-i \xi_{0} \frac{\partial}{\partial \xi_{2}}+\lambda \frac{\xi_{1}}{\xi_{0}+\xi_{3}}, \\
& N_{3}=-i \xi_{0} \frac{\partial}{\partial \xi_{3}} .
\end{aligned}
$$

For the parametrization (3.4) of $\xi$, the Casimir invariants have the form

$\mathbf{M}^{2}-\mathbf{N}^{2}=\frac{d^{2}}{d c^{2}}+2 \frac{d}{d c}+\lambda^{2}, \mathbf{M} \cdot \mathbf{N}=i \lambda\left(1+\frac{d}{d c}\right)$.

From (3.4) and (3.6) it is not hard to show that the simultaneous eigenfunctions of $\mathbf{M}^{2}-\mathrm{N}^{2}, \mathbf{M} \cdot \mathbf{N}$, $M_{3}$ and $N_{3}$ have the form 


$$
e_{p \lambda}(\tau, \rho)=e^{-(1-i \rho) c} e^{i p \phi} e^{i \tau \beta} e^{-i \lambda \phi},
$$

in particular, on the $C$ system contour

$$
\mathcal{C}_{p \lambda}(\tau, \rho)=e^{i p \phi} e^{i \tau \beta} e^{-i \lambda \phi} .
$$

The function $\Phi_{j_{0} \rho}(\xi)$ is now expanded in terms of the $\mathcal{C}_{p \lambda}(\tau, \rho)$ functions according to

$$
\Phi_{j_{0} \rho}(\xi)=\sum a_{p, \tau}^{j_{0}}(\tau, \rho) \mathcal{C}_{p j_{0}}(\tau, \rho) .
$$

For evaluation of the integral over $d^{2} \xi$ in (3.1), it is most convenient to assume $u$ in the form

$$
u=u_{0}=(\cosh a, \sinh a, 0,0)
$$

the required expansion for the more general form of $u$ can be obtained by using the simple group properties of the $O(1,1) \otimes O(2)$ matrix elements. So combining (3.9) and (3.1) requires the calculation of the following integral:

$I=\int_{0}^{2 \pi} d \phi \int_{-\infty}^{\infty} d \beta(\cosh a \cosh \beta-\sinh a \cos \phi)^{-1-i \rho}$

$$
\times D_{\lambda j_{0}}^{s}(R) \mathbb{e}_{p j_{0}}(\tau, \rho) \text {. }
$$

We now turn our attention to the explicit form of $D_{\lambda j_{0}}^{s}(R)$. For this it is convenient to write

$$
\mathbf{n}=((\cos \phi / \cosh \beta),(\sin \phi / \cosh \beta), \tanh \beta),
$$

the direction vector of the photon 3-momentum. Now if $\mathbf{n}$ is rotated by $-\phi$ about the $z$ axis, $\mathbf{n}$ becomes

$$
\mathrm{n} \rightarrow \mathrm{n}_{0}=((1 / \cosh \beta), 0, \tanh \beta) .
$$

According to the prescription of Ref. 7, the remaining rotation is a rotation in the $x z$ plane by an amount $\eta$ given by

$$
\cos \eta=\frac{u_{0} \cos \theta-|\mathbf{u}|}{u_{0}-\mathbf{u} \cdot \mathbf{n}},
$$

where $\theta$ is the angle between $\mathbf{n}_{\mathbf{0}}$ and $\mathbf{u}=($ sha, 0,0$)$. In our case

$$
\cos \theta=1 / \cosh \beta
$$

and

$$
\cos \eta=\frac{\cosh a-\cosh \beta \sinh a}{\cosh a \cosh \beta-\sinh a}
$$

so that we finally have

$$
R=M_{3}\left(\frac{1}{2} \pi-\phi\right) M_{1}(\eta) M_{3}\left(-\frac{1}{2} \pi\right) .
$$

The integral $I$ can now be evaluated. It is found to be given by

$$
\begin{aligned}
I= & \frac{e^{i \pi\left(\lambda-j_{0}\right)}}{\Gamma(1+i \rho)} \sum_{r_{i}} \frac{\Gamma\left(1+2 r_{1}+i \rho\right)}{\Gamma\left(r_{1}+1-\frac{1}{2} \bar{p}\right) \Gamma\left(r_{1}+1+\frac{1}{2} \bar{p}\right)} \\
& \times \Lambda_{s r_{4}, \lambda j_{0}} \frac{\left(2 r_{1}+1+i \rho\right)_{r_{2}}(-i \tau)_{r_{3}}\left(-i \tau+\frac{1}{2}\right) r_{3}}{\left(\frac{1}{2}\right)_{r_{3}} r_{2} ! r_{3} !} \\
& \times \frac{\Gamma(s) \Gamma(c-b)}{\Gamma(c)} e^{a\left(\lambda-j_{0}+2 r_{4}\right)} \\
& \times\left(\frac{1}{2} \tanh a\right)^{2 r_{1}}(\cosh a)^{-1-i \rho}{ }_{2} F_{1}\left(s, b ; c ;-e^{2 a}\right),
\end{aligned}
$$

where

$$
\begin{aligned}
& b= \frac{1}{2}\left(\lambda-j_{0}\right)+r_{2}+r_{3}+r_{4}+\frac{1}{2}, \\
& c= 2 r_{1}+i \rho-i \tau+\frac{1}{2}\left(\lambda-j_{0}\right)+r_{2}+r_{3}+r_{4}+\frac{3}{2}, \\
& \Lambda_{s r_{4}, \lambda j_{n}}= {\left[\Gamma(s+\lambda+1) \Gamma(s-\lambda+1) \Gamma\left(s+j_{0}+1\right)\right.} \\
&\left.\times \Gamma\left(s-j_{0}+1\right)\right]^{1 / 2}\left[\Gamma\left(s-\lambda-j_{0}+1\right)\right. \\
& \times \Gamma\left(s+j_{0}-r_{4}+1\right) \Gamma\left(r_{4}+\lambda-j_{0}+1\right) \\
&\left.\times \Gamma\left(r^{4}+1\right)\right]^{-1} \\
&(d)_{n}=\Gamma(d+n) / \Gamma(d), \quad \bar{p}=p-\lambda .
\end{aligned}
$$

We now identify $I$ with the mixed basis matrix element in the following way:

$$
\left\langle\rho j_{0} ; s \lambda\left|N_{1}(a)\right|_{\rho j_{0}} ; \tau p\right\rangle=C_{s \lambda, \tau p}^{\rho j_{0}}(a)=I .
$$

The expansion of a single particle helicity state in terms of $C$ system matrix elements is then

$$
\begin{aligned}
& |u ; s, \lambda\rangle=\frac{1}{2(2 \pi)^{3}} \sum_{j_{0}=-s}^{s} \int_{-\infty}^{\infty} d \rho\left(\rho^{2}+j_{0}^{2}\right) \\
& \quad \times \sum_{p=-\infty}^{\infty} \int_{-\infty}^{\infty} d \tau a_{p}^{j_{0}}(\tau, \rho) C_{s \lambda_{;} ; M}^{\rho j_{0}}(a) e^{i \tau b} e^{i p \psi} .
\end{aligned}
$$

\section{RECURRENCE RELATIONS FOR THE MIXED BASIS MATRIX ELEMENTS}

In this section we use the infinitesmal operator method 10.11 to establish recurrence relations and differential equations for the mixed basis matrix elements. For this method we use a fixed column of the mixed basis matrix element $\left\langle\rho j_{0} ; J M|L| \rho j_{0} ; \tau p\right\rangle$ (i.e., $\tau$ and $p$ fixed) as a set of $S U(2)$ basis vectors spanning the UIR $\left\{j_{0}, \rho\right\}$ of $S L(2, C) . L$ is a general lorentz transformation. The generators $M_{i}, N_{i}$ are then differential operators acting on the six parameters needed to specify $L$. Now using Eqs. (1.10) and (1.9) and making a particular choice for $L$ we can derive the relations we need. For the $C$ system we parametrize $L$ as follows

$$
L=M_{3}(\phi) M_{1}(\theta) M_{3}(\alpha) N_{1}(a) N_{3}(b) M_{3}(\psi),
$$

so that the mixed basis matrix element is

$$
\begin{aligned}
& \left\langle\rho j_{0} ; J M|L| \rho j_{0} ; \tau p\right\rangle=\bar{C}_{J M, \tau p}^{\rho j_{0}} \\
& \quad=\sum_{\lambda} D_{M \lambda}^{J}(\phi, \theta, \alpha) C_{J \lambda, \tau p}^{\rho j_{0}}(a) e^{i \tau b} e^{i p \psi} .
\end{aligned}
$$

The generators $M_{i}, N_{i}$ corresponding to the parametrization (4.1) are

$M_{1}=-\cot \theta \sin \phi \frac{\partial}{\partial \phi}+\cos \phi \frac{\partial}{\partial \phi}+\frac{\sin \phi}{\sin \theta} \frac{\partial}{\partial \alpha}$, $M_{3}=\frac{\partial}{\partial \phi}$,

$N_{1}=-\sin \phi \cos \alpha \tanh a \frac{\partial}{\partial \phi}+\tanh a \sin \phi \sin \theta \sin \alpha \frac{\partial}{\partial \theta}$ $-\frac{1}{\sinh a \cosh a}\left(\sin \phi \cos \theta \cos \alpha+\cos \phi \sin \alpha \cosh ^{2} a\right)$ $\times \frac{\partial}{\partial \alpha}+(\cos \phi \cos \alpha-\sin \phi \sin \alpha \cos \theta) \frac{\partial}{\partial a}+\frac{\sin \phi \sin \theta}{\cosh a}$ $\times \frac{\partial}{\partial b}+\frac{1}{\sinh a}(\cos \phi \sin \alpha+\sin \phi \cos \alpha \cos \theta) \frac{\partial}{\partial \psi}$, $N_{3}=-\cot \theta \cos \alpha \tanh a \frac{\partial}{\partial \phi}+\tanh a \cos \theta \sin \alpha \frac{\partial}{\partial \theta}$ $+\cos \alpha(\tanh a \cot \theta \cos \theta+\sin \theta \operatorname{coth} a) \frac{\partial}{\partial a}$ $+\sin \theta \sin \alpha \frac{\partial}{\partial \alpha}$. 
$M_{2}$ and $N_{2}$ can be obtained from $M_{1}$ and $N_{1}$, respectively, via the transformation $\phi \rightarrow-\frac{1}{2} \pi+\phi$.

In the $S U(2)$ basis we have chosen, the Casimir invariant equations have the form

$$
\begin{aligned}
\left(\mathbf{M}^{2}-\mathbf{N}^{2}\right) \bar{C}_{J M, \tau p}^{\rho j_{0}}= & \left(1+\rho^{2}-j_{0}^{2}\right) \bar{C}_{J M, \tau p}^{\rho j_{0}}, \\
& \mathbf{M} \cdot \mathbf{N} \bar{C}_{J M, \tau p}^{\rho j_{0}}=\rho j_{0} \bar{C}_{J M, \tau p}^{\rho j_{0}} .
\end{aligned}
$$

The explicit expression of the Casimir invariants in terms of differential operators is found from (4.3) to be

$$
\begin{aligned}
\mathbf{N}^{2}-\mathbf{M}^{2}= & \frac{\partial^{2}}{\partial a^{2}}+(\tanh a+\operatorname{coth} a) \frac{\partial}{\partial a}+\frac{1}{\cosh ^{2} a} \frac{\partial^{2}}{\partial b^{2}} \\
& +\frac{1}{\sinh ^{2} a} \frac{\partial^{2}}{\partial \psi^{2}}-2 \frac{\operatorname{coth} a}{\sinh a} \frac{\partial^{2}}{\partial \alpha \partial \psi} \tanh ^{2} a \bar{M}_{2}^{2} \\
& -\mathbf{M}^{2}+2 \frac{\tanh a}{\cosh a} \bar{M}_{2} \frac{\partial}{\partial b}+\operatorname{coth}^{2} a \frac{\partial^{2}}{\partial \alpha^{2}}
\end{aligned}
$$

$$
\begin{aligned}
\mathrm{M} \cdot \mathrm{N}=\bar{M}_{1}\left(\frac{\partial}{\partial a}+\right. & \tanh a)+\bar{M}_{2}\left((\tanh a-\operatorname{coth} a) \frac{\partial}{\partial \alpha}\right. \\
& \left.+\frac{1}{\sinh a} \frac{\partial}{\partial \psi}\right)+\frac{1}{\operatorname{coth} a} \frac{\partial^{2}}{\partial b \partial \alpha}
\end{aligned}
$$

where

$$
\bar{M}_{2}=\cot \theta \cos \alpha \frac{\partial}{\partial \alpha}+\sin \alpha \frac{\partial}{\partial \theta}-\frac{\cos \alpha}{\sin \theta} \frac{\partial}{\partial \phi},
$$

$$
\bar{M}_{1}=-\cot \theta \sin \alpha \frac{\partial}{\partial \alpha}+\cos \alpha \frac{\partial}{\partial \theta}+\frac{\sin \alpha}{\sin \theta} \frac{\partial}{\partial \phi} .
$$

So applying the Casimir invariants $(4.5)$ to the $\bar{C}_{J M, \tau p}^{\rho j_{0}}$ functions and separating out all but the $a$ dependence, using known recurrence relations of the $S U(2)$ matrix elements 12 and the orthogonality properties of the $O(1,1) \otimes O(2)$ matrix elements, we get the relations

$$
\begin{aligned}
& \alpha_{\lambda}^{J}\left(\frac{d}{d a}+\lambda \tanh a+(1-\lambda) \operatorname{coth} a+\frac{p}{\sinh a}\right) C_{J, \lambda+1 ; \tau p}^{\rho j_{0}} \\
& +\alpha_{\lambda+1}^{J}\left(\frac{d}{d a}-\lambda \tanh a+(1+\lambda) \operatorname{coth} a-\frac{p}{\sinh a}\right) \\
& \quad \times C_{J, \lambda-1 ; \tau p}^{\rho j_{0}}+i\left(\frac{2 \lambda \tau}{\cosh a}+\rho j_{0}\right) C_{J \lambda ; \tau p}^{\rho j_{0}}=0 \\
& \left(\frac{d^{2}}{d a^{2}}+(\tanh a+\operatorname{coth} a) \frac{d}{d a}-\frac{\tau^{2}}{\cosh ^{2} a}-\frac{p^{2}}{\sinh ^{2} a}\right. \\
& +2 \lambda p \frac{\operatorname{coth} a}{\sinh a}+J(J+1)+\frac{1}{2} \tanh ^{2} a[J(J+1) \\
& \left.-\lambda^{2} \operatorname{coth}^{2} a+\left(1-j_{0}^{2}+\rho^{2}\right)\right) C_{J \lambda ; \tau p}^{\rho j 0} \\
& +\frac{1}{4} \tanh ^{2} a\left[\alpha_{\lambda+1}^{J} \alpha_{\lambda+2}^{J} C_{J, \lambda+2 ; \tau p}^{\rho j_{0}}+\alpha_{\lambda}^{J} \alpha_{\lambda-1}^{J} C_{J, \lambda-2 ; \tau p}^{\rho j_{0}}\right] \\
& +i \tau(\tanh a / \cosh a)\left(\alpha_{\lambda}^{J} C_{J, \lambda-1 ; \tau p}^{\rho j_{0}}-\alpha_{\lambda+1}^{J} C_{J, \lambda+1 ; \tau p}^{\rho j_{0}}\right)=0 .
\end{aligned}
$$

The remaining recurrence relations are determined from the known action of the generators $N_{ \pm}$in an $S U(2)$ basis [Eqs. $(1,10)]$. They are

$$
\begin{aligned}
& {[(J+\lambda)(J+\lambda+1)]^{1 / 2}\left(\frac{d}{d a}+\frac{p}{\sinh a}+(1-\lambda) \operatorname{coth} a+(J-\lambda+1) \tanh a\right) C_{J, \lambda-1 ; \tau p}^{\rho j_{0}} } \\
&-[(J-\lambda)(J-\lambda+1)]^{1 / 2}\left(\frac{d}{d a}-\frac{p}{\sinh a}+(1+\lambda) \operatorname{coth} a+(J+\lambda+1) \tanh a\right) C_{J, \lambda+1 ; \tau p}^{\rho j_{0}} \\
&-(2 i \tau / \cosh a)\left[(J+1)^{2}-\lambda^{2}\right]^{1 / 2} C_{J \lambda, \tau p}^{\rho j_{0}} \\
&=2\left\{\left[(J+1)^{2}-j_{0}^{2}\right]\left[(J+1)^{2}+\rho^{2}\right][(2 J+1) /(2 J+3)]\right\}^{1 / 2} C_{J+1, \lambda ; \tau p}^{\rho j_{0}} \\
&-[(J-\lambda)(J-\lambda+1)]^{1 / 2}\left(\frac{d}{d a}+\frac{p}{\sinh a}+(1-\lambda) \operatorname{coth} a+(J+\lambda) \tanh d\right) C_{J, \lambda-1 ; \tau p}^{\rho j_{0}}+[(J+\lambda)(J+\lambda+1)]^{1 / 2} \\
& \times\left(\frac{d}{d a}-\frac{p}{\sinh a}+(1+\lambda) \operatorname{coth} a+(J-\lambda) \tanh a\right) C_{J, \lambda+1 ; \tau p}^{\rho j_{0}}+\left(J^{2}-\lambda^{2}\right)^{1 / 2}(2 i \tau / \cosh a) C_{J \lambda ; \tau p}^{\rho j_{0}} \\
&= 2\left\{\left(J^{2}-j_{0}^{2}\right)\left(J^{2}+\rho^{2}\right)[(2 J+1) /(2 J-1)]\right\}^{1 / 2} C_{J-1 ; \lambda, \tau p}^{\rho j_{0}}
\end{aligned}
$$

These relations we have developed here are the ones we will use in the next section in our analysis of the Proca and Dirac fields.

\section{SOLUTION OF THE DIRAC AND PROCA FREE FIELD EQUATIONS IN THE $C$ SYSTEM}

As an application of the previous three sections we derive invariant expansions of solutions of the Dirac and Proca equations in terms of the functions

$$
D_{J \lambda ; \tau p}^{\rho j_{0}}(a, b, \phi)=C_{J \lambda ; \tau p}^{\rho j_{0}}(a) e^{i \tau b} e^{i p \phi} \text {. }
$$

This has already been done in the $S$ system for these equations ${ }^{13}$ and more general ones. ${ }^{14,15}$

An outline of the general method is as follows. In order to achieve an invariant expansion of an arbitrary field $F_{J M}^{\rho j_{0}}(x)$, it is convenient to go over into a coordinate system in which each component transforms independently. The components of $F_{J M}{ }^{\rho j_{0}}(x)$ in this new coordinate system are
$\bar{F}_{J M}^{\rho j_{0}}(g)=U(g) F_{J M}^{\rho j_{0}}(x)=D_{J^{\prime} M^{\prime}, J M}^{\rho j_{0}}(g) F_{J^{\prime} M^{\prime}}^{\rho j_{0}}\left(g^{-1} x\right)$

From this definition it follows that each component does indeed transform independently:

$$
U\left(g_{0}\right) \bar{F}_{J M}^{\rho j_{0}}(g)=\bar{F}_{J M}^{\rho j_{0}}\left(g_{0} g\right)
$$

so that each component of $\bar{F}_{J M}^{\rho j_{0}}(g)$ constitutes a representation space for the Lorentz group and can, therefore, be expanded in terms of matrix elements of that group.

We now turn our attention to the Proca field $A_{K}(x)$ of mass $\mu$, i.e.,

$$
\begin{aligned}
\left(\square-\mu^{2}\right) A_{K}(x)=\left(\frac{\partial^{2}}{\partial x_{1}{ }^{2}}\right. & \left.+\frac{\partial^{2}}{\partial x_{2}{ }^{2}}+\frac{\partial^{2}}{\partial x_{3}{ }^{2}}-\frac{\partial^{2}}{\partial x_{0}{ }^{2}}-\mu^{2}\right) \\
& \times A_{K}(x)=0, \frac{\partial A_{K}}{\partial x_{K}}=0 .
\end{aligned}
$$

We seek a solution for this equation inside the light 
cone, so in the $C$ system we choose $x$ to be parametrized by

$x=(s \cosh a \cosh b, s \sinh a \cos \phi, s \sinh a \sin \phi$, $s \cosh a \sinh b)$.

The operators $\partial / \partial x_{i}$ have the form

$\frac{\partial}{\partial x_{0}}=\cosh a \cosh b \frac{\partial}{\partial s}-\frac{\sinh a \cosh b}{s} \frac{\partial}{\partial a}-\frac{\sinh b}{s \cosh a} \frac{\partial}{\partial b}$, $\frac{\partial}{\partial x_{1}}=-\sinh a \cos \phi \frac{\partial}{\partial s}+\frac{\cosh a \cos \phi}{s} \frac{\partial}{\partial a}-\frac{\sin \phi}{s \sinh a} \frac{\partial}{\partial \phi}$, $\frac{\partial}{\partial x_{2}}=-\sinh a \sin \phi \frac{\partial}{\partial s}+\frac{\cosh a \sin \phi}{s} \frac{\partial}{\partial a}+\frac{\cos \phi}{s \cosh a} \frac{\partial}{\partial b}$ $\frac{\partial}{\partial x_{3}}=-\sinh b \cosh a \frac{\partial}{\partial s}+\frac{\sinh a \sinh b}{s} \frac{\partial}{\partial a}$

$$
+\frac{\cosh b}{s \cosh a} \frac{\partial}{\partial b}
$$

The transformation to the independent variables changes the 4-vector $x$ as if at the point $(a, b, \phi)$ the space has been subjected to the Lorentz transformation

$$
\Omega=N_{1}(-a) N_{3}(-b) M_{3}(-\phi) .
$$

Under this transformation $\partial / \partial x_{K}$ and $A_{K}(x)$ are transformed according to

$$
\frac{\partial}{\partial \bar{x}_{K}}=\Omega_{k n} \frac{\partial}{\partial x_{n}}, \quad \bar{A}_{K}(\bar{x})=\Omega_{k t} A_{l}(x)
$$

where

$$
\begin{aligned}
& \frac{\partial}{\partial \bar{x}_{0}}=\frac{\partial}{\partial s}, \quad \frac{\partial}{\partial \bar{x}_{1}}=\frac{1}{s} \frac{\partial}{\partial a}, \\
& \frac{\partial}{\partial \bar{x}_{2}}=\frac{1}{s \sinh a} \frac{\partial}{\partial \phi}, \quad \frac{\partial}{\partial \bar{x}_{3}}=\frac{1}{s \cosh a} \frac{\partial}{\partial b},
\end{aligned}
$$

The transformed Proca equation now becomes

$$
\begin{aligned}
& \left(\square-\mu^{2}\right) \bar{A}_{k}(\bar{x})-D_{i} \frac{\partial \bar{A}_{k}(\bar{x})}{\partial \bar{x}_{i}}-\Omega_{k l} \\
& \quad \times\left(D_{i} \frac{\partial \Omega_{l v}^{-1}}{\partial \bar{x}_{i}}+2 \frac{\partial \Omega_{l v}^{-1}}{\partial \bar{x}_{i}} \frac{\partial}{\partial \bar{x}_{i}}+\frac{\partial^{2} \Omega_{l v}^{-1}}{\partial \bar{x}_{i}^{2}}\right) \bar{A}_{\nu}(\bar{x})=0 \\
& \quad \frac{\partial \bar{A}_{i}(\bar{x})}{\partial \bar{x}_{i}}+D_{i} \bar{A}_{i}(\bar{x})=0
\end{aligned}
$$

where

$$
D_{i}=\frac{\partial \Omega_{i \nu}}{\partial \bar{x}_{k}} \Omega_{\nu k}^{-1}
$$

passing to the canonical basis

$$
f_{0}=A_{0}, \quad \sqrt{ } 2 f_{ \pm}=i A_{2} \mp A_{1}, \quad f_{1}=A_{3}
$$

and expanding $f_{0}, f_{1}$ and $f_{ \pm}$according to

$$
\begin{aligned}
& f_{0}=\sum \chi_{0}^{\left(\rho, j_{0}\right)}(s) C_{00 ; \tau p}^{\rho j_{0}} e^{i \tau b} e^{i p \phi}, \\
& f_{ \pm}=\sum \chi_{t}^{\left(\rho, j_{0}\right)}(s) C_{1, \pm 1 ; \tau p}^{\rho j_{0}} e^{i \tau b} e^{i p \phi}, \\
& f_{1}=\sum \chi_{1}^{\left(\rho, j_{0}\right)}(s) C_{1,0 ; \tau p} e^{i \tau b} e^{i p \phi},
\end{aligned}
$$

where the summation is over $j_{0}, p, \tau, \rho$, the system of equations (5.10) becomes

$$
\begin{aligned}
& \left(\frac{\partial \chi_{0}}{\partial s}+\frac{3}{s} \chi_{0}\right) C_{0}+\frac{1}{\sqrt{2 s}}\left[\left(\frac{\partial C_{-}}{\partial a}+(\tanh a+\operatorname{coth} a) C_{-}+\frac{p}{\sinh a} C_{-}\right) x_{-}\right. \\
& \left.-\left(\frac{\partial C_{+}}{\partial a}+(\tanh a+\operatorname{coth} a) C_{+}-\frac{p}{\sinh a} C_{+}\right) \chi_{+}+\frac{\sqrt{2} i \tau}{\cosh a} \chi_{1} C_{1}\right]=0, \\
& \left(\frac{\partial^{2} \chi_{0}}{\partial s}+\frac{3}{s} \frac{\partial \chi_{0}}{\partial s}-\frac{3 \chi_{0}}{s^{2}}+u^{2} \chi_{0}\right) C_{0}-\frac{1}{s^{2}}\left[\left(\frac{\partial^{2} C_{0}}{\partial a^{2}}+(\tanh a+\operatorname{coth} a) \frac{\partial C_{0}}{\partial a}\right.\right. \\
& \left.-\frac{\tau^{2}}{\cosh ^{2} a} C_{0}-\frac{p^{2}}{\sinh ^{2} a} C_{0}\right) \chi_{0}+\sqrt{2}\left(\frac{\partial C_{-}}{\partial a}+(\tanh a+\operatorname{coth} a) C_{-}+\frac{p}{\sinh a} C_{-}\right) \chi_{-} \\
& \left.-\sqrt{2}\left(\frac{\partial C_{+}}{\partial a}+(\tanh a+\operatorname{coth} a) C_{+}-\frac{p}{\sinh a} C_{+}\right) \chi_{+}+\frac{2 i \tau}{\cosh a} x_{1} C_{1}\right]=0, \\
& \left(\frac{\partial^{2} \chi_{1}}{\partial s^{2}}+\frac{3}{s} \frac{\partial X_{1}}{\partial s}+\mu^{2} \chi_{1}\right) C_{1}-\frac{1}{s^{2}}\left[\left(\frac{\partial^{2} C_{1}}{\partial a^{2}}+(\tanh a+\operatorname{coth} a) \frac{\partial C_{1}}{\partial a}-\frac{\tau^{2}}{\operatorname{coth}^{2} a} C_{1}\right.\right. \\
& \left.\left.-\frac{p^{2}}{\sinh ^{2} a} C_{1}+\frac{1}{\cosh ^{2} a} C_{1}\right) \mathrm{x}_{1}+i \tau \sqrt{2} \frac{\tanh a}{\cosh a}\left(\mathrm{x}_{-} C_{-}-\mathrm{x}_{+} C_{+}\right)+\frac{2 i \tau}{\cosh a} \mathrm{x}_{0} C_{0}\right]=0 \text {, } \\
& \left(\frac{\partial^{2} \chi_{ \pm}}{\partial s^{2}}+\frac{3}{s} \frac{\partial \chi_{ \pm}}{\partial s}+\mu^{2} \chi_{ \pm}\right) C_{ \pm}-\frac{1}{s^{2}}\left[\left(\frac{\partial^{2} C_{ \pm}}{\partial a^{2}}+(\tanh a+\operatorname{coth} a) \frac{\partial C_{ \pm}}{\partial a}-\frac{\tau^{2}}{\cosh ^{2} a} C_{ \pm}-\frac{p}{\sinh ^{2} a} C_{ \pm}-\frac{1}{\sinh ^{2} a} C_{ \pm}\right.\right. \\
& \left.\left.-\frac{1}{2} \tanh ^{2} a C_{ \pm} \pm 2 p \frac{\operatorname{coth} a}{\sinh a} C_{t}\right) \chi_{ \pm} \pm i \tau \sqrt{2} \frac{\tanh a}{\cosh a} x_{1} C_{1}+\frac{1}{2} \tanh ^{2} a \chi_{\mp} C_{\mp}+2\left(\mp \frac{\partial C_{0}}{\partial a}-\frac{p}{\sinh a} C_{0}\right) \chi_{0}\right]=0 \text {, }
\end{aligned}
$$

where we have used the shorthand

$$
C_{0}=C_{00 ; \tau \rho}^{\rho j_{0}}, \quad C_{ \pm}=C_{1, \pm 1 ; \tau p}^{\rho j_{0}}, \quad C_{1}=C_{1,0 ; \tau p}^{\rho j_{0}} .
$$

From the recurrence relations (4.7)-(4.10) we see that the variables separate if

$$
x_{+}=x_{-}=-x_{1}
$$

We then arrive at the same system of equations as in Ref. 13 viz.

$$
\left(\frac{d}{d s}+\frac{3}{s}\right) x_{0}^{(\rho, 0)}(s)+\frac{\left[3\left(1+\rho^{2}\right)\right]^{1 / 2}}{s} \chi_{1}^{(\rho, 0)}(s)=0,
$$

J. Math. Phys., Vol. 13, No. 9, September 1972 


$$
\begin{aligned}
& \left(\frac{d}{d s^{2}}+\frac{5}{s} \frac{d}{d s}+\frac{4+\rho^{2}}{s^{2}}+\mu^{2}\right) x_{0}^{(\rho, 0)}=0, \\
& \left(\frac{d^{2}}{d s^{2}}+\frac{3}{s} \frac{d}{d s}+\frac{1+\rho^{2}}{s^{2}}+\mu^{2}\right) \chi_{1}^{(\rho \pm 1)}(s)=0
\end{aligned}
$$

(remember the summation on $j_{0}$ consists of $j_{0}=0$ only, for $f_{0}$ ). These equations have the solution

$$
\begin{aligned}
& \chi_{1}^{(\rho, \pm 1)}(s)=(1 / \mu s)\left[c_{1} H_{i \rho}^{(2)}(\mu s)+c_{2} H_{-i \rho}^{(2)}(\mu s)\right], \\
& \chi_{0}^{(\rho, 0)}(s)=\left[1 /(\mu s)^{2}\right]\left[c_{3} H_{i \rho}^{(2)}(\mu s)+c_{4} H_{-i \rho}^{(2)}(\mu s)\right] .
\end{aligned}
$$

So the solutions to the Proca equation have the form

$f_{0}=\sum_{p=-\infty}^{\infty} \int_{-\infty}^{\infty} d \tau \int_{0}^{\infty} d \rho \chi_{0}^{(\rho, 0)}(s) C_{00, \tau p}^{\rho 0} e^{i \tau b} e^{i p \phi}$

$h_{\lambda}=\sum_{j_{0}=-1}^{+1} \sum_{p=-\infty}^{+\infty} \int_{0}^{\infty} d \tau \int_{0}^{\infty} d \rho \chi_{1}^{\left(\rho, j_{0}\right)}(s) C_{1, \lambda ; \tau p}^{\rho j_{0}} e^{i \tau b} e^{i p \phi}$,

where $h_{ \pm 1}=-f_{ \pm}, h_{0}=f_{1}$.

This then completes the derivation of an invariant expansion of solutions of the Proca equation inside the light cone.

We now turn our attention to the Dirac equation. In order to obtain an invariant decomposition of a solution of the Dirac equation, we write the equation in a canonical basis

$$
\left(i \gamma^{n} \frac{\partial}{\partial x^{n}}-\mu\right) \psi(x)=0
$$

where

$$
\gamma^{0}=\left(\begin{array}{ll}
0 & I \\
I & 0
\end{array}\right), \quad \gamma^{\alpha}=\left(\begin{array}{cc}
0 & -\sigma_{\alpha} \\
\sigma_{\alpha} & 0
\end{array}\right),
$$

( $\alpha=1,2,3)$, where $\sigma_{\alpha}$ are the Pauli spin matrices and $I$ the $2 \times 2$ identity matrix. Under the transformation $\Omega$ of (5.7), Eq. (5.18) changes to

$$
i \gamma^{n} \frac{\partial \bar{\psi}(\bar{x})}{\partial \bar{x}^{n}}+i \gamma^{n} \Lambda \frac{\partial \Lambda^{-1}}{\partial \bar{x}^{n}} \bar{\psi}(\bar{x})-\mu \bar{\psi}(\bar{x})=0,
$$

where $\bar{\psi}(\bar{x})=\Lambda \psi(x)$, i.e., $\Lambda$ is the $4 \times 4$ matrix according to which the spinor $\psi$ transforms under the

Lorentz transformation $\Omega$.

In the $C$ system we have that

$$
\gamma^{n} \Lambda \frac{\partial \Lambda^{-1}}{\partial \vec{x}^{n}}=\frac{i}{2 s}\left[(\tanh a+\operatorname{coth} a) \gamma^{1}-3 \gamma^{0}\right] .
$$

If we now look for solutions of the form

$$
\begin{array}{ll}
\psi_{i}=\sum f_{i}(s) C_{i}(a) e^{i \tau b} e^{i p \phi}, & i=1,3, \\
\psi_{j}=\sum f_{j}(s) C_{j}(a) e^{i \tau b} e^{i p \phi}, & j=2,4,
\end{array}
$$

the system of equations (5.20) becomes

$i\left(\frac{\partial f_{1}}{\partial s}+\frac{3}{2 s} f_{1}\right) C_{1}+\frac{\tau}{s \cosh a} f_{1} C_{1}-\frac{i}{s}\left(\frac{\partial C_{2}}{\partial a}\right.$

$\left.+\frac{p}{\sinh a} C_{2}+\frac{1}{2}(\tanh a+\operatorname{coth} a) C_{2}\right) f_{2}-\mu f_{3} C_{3}=0$,

$i\left(\frac{\partial f_{2}}{\partial s}+\frac{3}{2 s} f_{2}\right) C_{2}-\frac{\tau}{s \cosh a} f_{2} C_{2}-\frac{i}{s}\left(\frac{\partial C_{1}}{\partial a}\right.$

$\left.-\frac{p}{\sinh a} C_{1}+\frac{1}{2}(\tanh a+\operatorname{coth} a) C_{1}\right) f_{1}-\mu f_{4} C_{4}=0$, $i\left(\frac{\partial f_{3}}{\partial s}+\frac{3}{2 s} f_{3}\right) C_{3}-\frac{\tau}{s} \frac{\tau}{\cosh a} f_{3} C_{3}+\frac{i}{s}\left(\frac{\partial C_{4}}{\partial a}\right.$

$\left.+\frac{p}{\sinh a} C_{4}+\frac{1}{2}(\tanh a+\operatorname{coth} a) C_{4}\right) f_{4}-\mu f_{1} C_{1}=0$, $i\left(\frac{\partial f_{4}}{\partial s}+\frac{3}{2 s} f_{4}\right) C_{4}+\frac{\tau}{s \cosh a} f_{4} C_{4}+\left(\frac{\partial C_{3}}{\partial a}-\frac{p}{\sinh a} C_{3}\right.$ $\left.+\frac{1}{2}(\tanh a+\operatorname{coth} a) c_{3}\right) f_{3}-\mu f_{2} C_{2}=0$,

from which we see that the variables separate if we take

$$
\begin{aligned}
& f_{1}(s)=f_{2}(s), \quad f_{3}(s)=f_{4}(s), \\
& C_{i}(a)=C_{1 / 2,1 / 2 ; \uparrow p}^{\rho j_{0}}(a), \quad i=1,3, \\
& C_{j}(a)=C_{1 / 2,-1 / 2 ; \uparrow p}^{\rho j_{0}}(a), \quad j=2,4 .
\end{aligned}
$$

The form of $f_{1}(s)$ and $f_{3}(s)$ is now determined by the pair of coupled equations

$$
\begin{aligned}
& \left(\frac{d}{d s}+\frac{3}{2 s}-2 i j_{0} \rho\right) f_{3}+i \mu f_{1}=0, \\
& \left(\frac{d}{d s}+\frac{3}{2 s}+2 i j_{0} \rho\right) f_{1}+i \mu f_{3}=0,
\end{aligned}
$$

which have solutions of the for $\mathrm{m}^{16}$

$$
\begin{aligned}
& f_{1}(s)=(1 / \mu s)\left[c_{1} J_{\nu}(\mu s)+c_{2} J_{\nu}(\mu s)\right], \\
& f_{3}(s)=(1 / \mu s)\left[c_{2} J_{\nu}(\mu s)-c_{1} J_{-\nu}(\mu s)\right]
\end{aligned}
$$

with $\nu=\frac{1}{2}+2 i j_{0} \rho$.

So the solutions of the Dirac equation are

$$
\begin{aligned}
\psi_{i}=\sum_{j_{0}=-1 / 2}^{+1 / 2} \sum_{p=-\infty}^{\infty} \int_{-\infty}^{\infty} d \tau \int_{0}^{\infty} d \rho f_{i}(s) C_{1 / 2,1 / 2 ; \tau p}^{\rho j_{0}}(a) \\
\times e^{i \tau b} e^{i p \phi}, \quad i=1,3,
\end{aligned}
$$$$
\psi_{j}=\sum_{j_{0}=-1 / 2}^{+1 / 2} \sum_{p=-\infty}^{\infty} \int_{-\infty}^{\infty} d \tau \int_{0}^{\infty} d \rho f_{j}(s) C_{1 / 2,1 / 2 ; \tau p}^{\rho j_{0}}(a)
$$$$
\times e^{i \tau b} e^{i p \phi}, \quad j=2,4 .
$$

This then completes this section on the solution of the Proca and Dirac equation in the $C$ system.

\section{DIFFERENTIAL EQUATIONS SATISFIED BY THE EXPANSION MATRIX ELEMENTS AND THE NONRELATIVISTIC LIMIT}

From the recurrence relations derived in Sec. 4 we deduce that the matrix elements used in the expansions of Sec. 5 satisfy the following differential equations:

(i) Using the shorthand

$$
C_{J \lambda ; \tau p}^{p j_{0}}(a)=C_{J \lambda}^{\rho j_{0}}
$$

we have for $j_{0}=J=\lambda=0$ the differential equation

$$
\begin{aligned}
\left(\frac{d^{2}}{d a^{2}}+(\tanh a+\right. & \operatorname{coth} a) \frac{d}{d a}-\frac{\tau^{2}}{\cosh ^{2} a} \\
& \left.-\frac{p^{2}}{\sinh ^{2} a}\left(1+\rho^{2}\right)\right) C_{00}^{0}=0 .
\end{aligned}
$$


$C_{1 \lambda}^{0}$ may be calculated from $C_{00}^{0}$ by using

$$
\begin{aligned}
& -i \tau / \cosh a C_{00}^{0}=\left[\frac{4}{3}\left(1+\rho^{2}\right)-1 / 2 C_{10}^{0},\right. \\
& \quad \pm \sqrt{2}\left(\frac{d}{d a} \pm \frac{p}{\sinh a}\right) C_{00}^{0}=\left[\frac{4}{3}\left(1+\rho^{2}\right)\right]^{1 / 2} C_{1, \pm 1}^{0}
\end{aligned}
$$

(ii) $j_{0}=1 ; C_{10}^{1}$ satisfies the equation

$$
\begin{aligned}
& {\left[\frac{d^{2}}{d a^{2}}+\left((\tanh a+\operatorname{coth} a)+\frac{4 \tau}{\tau^{2}-\rho^{2} \cosh ^{2} a}\right) \frac{d}{d a}\right.} \\
& \quad-\frac{\tau^{2}}{\cosh ^{2} a}-\frac{p^{2}}{\sinh ^{2} a}+2+p^{2}+\tanh ^{2} a \\
& \left.+\frac{4 \tau \tanh a}{\tau^{2}-\rho^{2} \cosh ^{2} a}(2 \tau \tanh a-\rho p \operatorname{coth} a)\right] C_{10}^{1}=0 ;
\end{aligned}
$$

the other $j_{0}=1$ matrix elements may be deduced from the relations

$i\left(\mp \frac{2 \tau}{\cosh a}-\rho\right) C_{1, \pm 1}^{1}=\sqrt{2}\left(\frac{d}{d a}+\tanh a \pm \frac{p}{\sinh a}\right) C_{10}$.

(iii) $j_{0}=\frac{1}{2} ; C_{1 / 2,1 / 2}^{1 / 2}$ satisfies the equation

$$
\begin{aligned}
& {\left[\frac{d^{2}}{d a^{2}}+\left((\tanh a+\operatorname{coth} a)+\frac{\tau \tanh a}{\tau \pm \rho \cosh a}\right) \frac{d}{d a}-\frac{\tau^{2}}{\cosh ^{2} a}\right.} \\
& \quad-\frac{p^{2}}{\sinh ^{2} a} \pm p \frac{\operatorname{coth} a}{\sinh a}+\frac{1}{4}\left(\tanh ^{2} a-\operatorname{coth}^{2} a\right) \\
& \left.\quad+\frac{\tau}{\tau \pm \rho \cosh a}\left(\frac{1}{2}(\tanh a+\operatorname{coth} a) \mp \frac{p}{\sinh a}\right)+\rho^{2}\right] \\
& \quad \times C_{1 / 2, \mp 1 / 2}^{1 / 2}=0 .
\end{aligned}
$$

Similar equations to those of (ii) and (iii) hold for the cases $j_{0}=-1, j_{0}=-\frac{1}{2}$, respectively.

These equations are useful in the passage to the nonrelativistic limit.13-17 In this limit we have

$$
a \rightarrow 0, s \rightarrow \infty \quad \text { s.t. } \quad s a=r,
$$

where $r$ is the polar radius in the $x y$ plane in nonrelativistic 3 -space

$$
b \rightarrow 0, \quad s \rightarrow \infty \quad \text { s.t. } \quad s b=z .
$$

In addition we must require that

$$
\begin{aligned}
& \tau \rightarrow \infty \quad \text { in such a way that } \\
& \tau / s \rightarrow \tau^{\prime}, \quad-\infty<\tau^{\prime}<\infty ;
\end{aligned}
$$

finally

$$
\rho \rightarrow|\mathbf{p}| s .
$$

In this limit Eq. (6.1) becomes

$\left(\frac{d^{2}}{d r^{2}}+\frac{1}{r} \frac{d}{d r}+|\mathbf{p}|^{2}-\tau^{2}-\frac{p^{2}}{r^{2}}\right) C_{00}^{0}(r)=0$

so taking the regular solution at $r=0$, we have

$$
C_{00}^{0} \rightarrow c_{1} J_{p}(\alpha r), \quad \alpha^{2}=|\mathbf{p}|^{2}-\tau^{\prime 2} .
$$

From relations (6.2) we see that

$$
C_{10} \rightarrow c_{2} J_{p}(\alpha r), \quad C_{1, \pm 1}^{0} \rightarrow C_{ \pm} J_{p \pm 1}(\alpha r) .
$$

Similar results hold in the $j_{0}=1$ case as $C_{10}$ then satisfies Eq. (6.9)

This then gives the correct set of functions in 3space corresponding to the expansion of Maxwell's equations in cylindrical coordinates, 18 viz.,

$$
\begin{aligned}
& \bar{C}_{\lambda}(r, z, \phi)=J_{p^{+\lambda}}(\alpha r) e^{i \tau^{\prime} z} e^{i p \phi}, \\
& \lambda= \pm 1,0, \quad p=0, \pm 1, \pm 2, \ldots, \quad-\infty<\tau^{\prime}<\infty .
\end{aligned}
$$

We note that the solution in cylindrical coordinates is an expansion invariant with respect to the group $O(2) \otimes T_{3}$, the direct product of rotations about $O z$, and translations along $O z$. So the reduction $O(1,1) \otimes$ $O(2) \subset O(3,1)$ becomes in the nonrelativistic limit the reduction $O(2) \otimes T_{3} \subset E(3)$.

For the nonrelativistic limit of the functions used in the Dirac equation solution we have the following differential equations

$\left(\frac{d^{2}}{d r^{2}}+\frac{1}{r} \frac{d}{d r}+|\mathbf{p}|^{2}-\tau^{\prime 2}-\frac{\left(p \pm \frac{1}{2}\right)^{2}}{r^{2}}\right) c_{1 / 2, \pm 1 / 2}^{1 / 2}=0$

so that this corresponds to a nonrelativistic solution of the Dirac equation in terms of the complete set of functions

$$
P_{ \pm 1 / 2}(r, a, \phi)=J_{p \pm 1 / 2}(\alpha r) e^{i \tau^{\prime} z} e^{i p \phi} .
$$

This coincides with the solution in cylindrical coordinates in 3-space.

\section{CONCLUSION}

In this paper we have carried out the reduction of the principal series of $O(3,1)$ in an $O(1,1) \otimes O(2)$ basis and examined the properties of the $O(3) \leftrightarrow O(1,1) \otimes$ $O(2)$ mixed basis matrix elements. It was shown that the expansion of solutions of the Proca and Dirac free fields (inside the light cone) corresponds to the relativistic generalization of cylindrical coordinates in 3-space. In future developments we propose to study the solution of other wave equations (both inside and outside the light cone) using these mixed basis matrix elements. Other related problems of interest include the reduction of the supplementary series of $O(3,1)$ with respect to $O(1,1) \otimes O(2)^{19}$ and a study of the matrix elements in an $O(1,1) \otimes O(2)$ basis.

\section{ACKNOWLEDGMENTS}

The author would like to thank Professor I.A.Sakmar for his constant encouragement throughout the duration of this work. This work has been partially supported by the National Research Council research grant of Professor I. A. Sakmar for which we are grateful.
Work supported by a National Research Council of Canada graduate scholarship.

1 A partial list of references is the following: N. Mukunda, J, Math.
Phys. 9, 50, 417 (1968); K. Szegó and K. Tóth, ibid. 12, 846, 853 (1971); N. W. Macfadyen, ibid. 492 (1971); A. Sciarrino and M. Toller, ibid. 8, 1252 (1967); P.Winternitz, I. Lukač, and Ya. A. Smoro- 
dinski, Yad. Fiz.7, 192 (1968) [Sov.J.Nucl. Phys. 7, 139, (1968)]; S. Chang and L. O'Raifeartaigh, J. Math. Phys. 10, 21 (1969); Ya. A. Smorodinski and M.Huszar, Theoret. Math. Phys.4, $867(1970)$.

2 N. Ya. Vilenkin and Ya.A.Smorodinski, Zh. Eksp. Teor. Fiz.46, 1793 (1964); [Soviet Phys.JETP 19, 1209 (1964)].

3 For a discussion of Poincaré and Lorentz invariance in the scalar case, see P. Winternitz, Ya. A.Smorodinski, and M. B. Sheftel, Yad. Fiz. 7, 1325 (1968); [Sov. J. Nucl. Phys. 7, 785 (1969)].

4 In connection with little group Partial wave analysis (this is only a partial list), see H. Joos, Fortschr. Physik, 10,65 (1962); J.F Boyce, R. Delbourgo, A. Salam, and J. Strathdee, 1C/67/9 Trieste (1967); R. Delbourgo, A. Salam, and J.Strathdee, Phys. Letters 25B 230 (1967); M. Toller, Nuovo Cimento 53A, 671 (1968).

5 For a detailed treatment of the Lorentz group, see M.A. Naimark, Linear Representalions of the Lorentz Group (Pergamon, New York, 1964).

6 See I. M. Gel'fand and G. E. Shilov, Generalized Functions (Academic, New York, 1964), Vol.1, p.31.

7 G.I. Kuznetsov, M. A. Liberman, A. A. Makarov, and Ya. A. Smorodinski, Yad. Fiz. 10,644 (1969) [Sov.J. Nucl. Phys. 10, $370(1970)$ ]

s I. M. Gel'fand, M.I. Graev, and N. Ya. Vilenkin, Generalized Functions (Academic, New York, 1966), Vol. 5.
9 J.S. Lomont and Harry E. Moses, J. Math. Phys. 3, 405 (1963).

10 For details of this method in an $O(3)$ basis see $I$. A. Verdiev and L. A.Dadashev, Yad. Fiz.6, 1094 (1967) [Sov.J. Nucl. Phys.6, 795 (1968)].

11 S. Strom, Arkiv Fysik 29, 467 (1965).

12 I. M. Gel'fand, R. M. Minlos, and Z.Ya.Shapiro, Representations of the Rolation and Lorentz Groups and Their Applications (Pergamon, New York, 1963), p. 98.

13 A complete analysis of these equations in terms of $S U(2) \leftrightarrow$ $S U(1,1)$ mixed basis elements has been given by the author, E.G. Kalnins, in Ref. 18.

14 A. K. Agamaliev, N. M. Atakishiev, and I, A. Verdiev, Yad. Fiz.9, 201 (1969) [Sov.J. Nucl. Phys. 9, 120 (1969)] 10, 187 (1969) [10, 106 (1970)].

15 N. M. Atakishiev and I.A. Verdlev, Theoret. Math. Phys. 4, 833 (1970).

16 This agrees with Ref. 14.

17 V.G. Kadyshevsky, R.M. Mir-Kasimov, and N. B. Shakov, 1C /67/72, Trieste (1967).

18 E.G. Kalnins, Ph.D. thesis (University of Western Ontario).

19 For a treatment of the supplementary series reduced with respect to $O(2,1)$ see V.I. Kolomytsey, Theoret. Math. Phys . $2,153(1970)$.

\title{
Dynamics of Harmonically Bound Semi-Infinite and Infinite Chains with Friction and Applied Forces
}

\author{
K. H. Lee
}

Department of Physics, University of Missouri, Columbia, Missouri 65201

\author{
(Received 31 March 1972)
}

The dynamics of semi-infinite and infinite linear chains of identical masses and ideal springs is studied. In addition to the harmonic coupling between nearest neighbors, each particle is harmonically bound to ts equilibrium position and is subject to friction and time-dependent applied forces. The Laplace transform method is used to express the motion of all the particles. The exact solutions are found and discussed for four different cases: (a) an infinite chain, (b) a semi-infinite chain, (c) a semi-infinite chain with the position of the end particle specified as a function of time, and (d) an infinite chain with the position of one particle specified as a function of time. By specializing some results of the present work, those of previous calculations on simpler systems by other authors are recovered.

There are two main approaches to the mathematical description of physical phenomena. One sometimes tries to study as exactly as possible a simplified model with only the main features of a real system, while some are more interested in an approximate solution of a realistic model. The one-dimensional systems have been favorite models for the first approach. ${ }^{1}$ One such system extensively studied is the infinite chain of point masses and ideal massless springs ${ }^{1,2}$ because it is one of the very few manybody systems in which exact calculations are possible. However, there has not been much study of an exact treatment of a semi-infinite chain. Although there have been many calculations treating semiinfinite lattices in conjunction with studies on surface phenomena, ${ }^{3}$ most of them can be classified under the second approach above.

The present work studies the exact dynamics of semi-infinite and infinite linear chains of identical masses and ideal massless springs with identical force constants. In addition to the harmonic coupling between nearest neighbors, each mass is harmonically bound to its equilibrium position and is subject to friction and time-dependent applied forces. The motion of each of the particles is expressed exactly in terms of the given quantities and initial conditions. Four different systems are studied; (a) an infinite chain, (b) a semi-infinite chain, (c) a semi-infinite chain with the position of the end particle specified as a function of time, and (d) an infinite chain with the position of one particle specified as a function of time. By specializing some of the results, those of previous calculations on simpler systems by other authors are recovered.

Let $x_{n}(t)$ represent the displacement of the $n$th particle measured from its equilibrium position. The integer $n$ is restricted to $n \geq 0$ for the semiinfinite systems (b) and (c). The coupled equations for the system are

$$
\begin{aligned}
& m x_{n}=-k\left(x_{n}-x_{n+1}\right) \\
& -k\left(x_{n}-x_{n-1}\right)\left[\begin{array}{c}
1 \\
\left(1-\delta_{n 0}\right)
\end{array}\right]-K x_{n}-\beta \dot{x}_{n}+\phi_{n},
\end{aligned}
$$

where $m$ is the particle mass, $k$ and $K$ are the spring constants, $\beta$ is the friction coefficient, $\delta$ is the Kronecker delta, $\phi_{n}(t)$ represents the external force applied to the $n$th particle and is assumed to be a known function of time. This system of equations is to be solved for $x_{n}(t)$ subject to the initial conditions

$$
x_{n}(0)=a_{n}, \quad \dot{x}_{n}(0)=v_{n} .
$$

For cases (c) and (d), in which $x_{0}(t)$ is specified, Eq (1) for $n=0$ determines the applied force $\phi_{0}(t)$ required to achieve such a specified motion for the particle $n=0$.

If one assumes that $x_{n}$ and $\phi_{n}$ have the Laplace transforms

$$
\begin{aligned}
& X_{n}(s)=L\left\{x_{n}(t)\right\}=\int_{0}^{\infty} d t x_{n}(t) \exp (-s t), \\
& \Phi_{n}(s)=L\left\{\phi_{n}(t) / k\right\}
\end{aligned}
$$

then Eqs. (1) and (2) lead to an inhomogeneous linear difference equation of second order

$$
\begin{gathered}
X_{n+1}-2\left(2 \alpha^{2}+4 \mu \sigma+2 \alpha^{2}-(1 / 2)\left[\begin{array}{c}
0 \\
\delta_{n 0}
\end{array}\right]\right) X_{n} \\
+X_{n-1}\left[\begin{array}{c}
1 \\
\left(1-\delta_{n 0}\right)
\end{array}\right]=-H_{n},
\end{gathered}
$$

